\title{
Single-Frequency 389-nm Coherent Light by Efficient Wavelength Conversion for Magnetic Resonance Imaging of Porous Materials with Nuclear Spin Polarization of ${ }^{3}$ He Atoms
}

\author{
Shingo Maeda, Yutaka Tabata, Hiroshi Morioka Hiroshi Kumagai, and Ataru Kobayashi \\ Osaka City University, 3-3-138 Sugimoto, Sumiyoshi-ku, Osaka 558-8585, Japan \\ kumagai@a-phys.eng.osaka-cu.ac.jp
}

\begin{abstract}
We report that we developed the efficient $389-\mathrm{nm} \mathrm{cw}$ coherent light, corresponding to the optical transition of $2^{3} \mathrm{~S}_{1} \rightarrow 3^{3} \mathrm{P}_{\mathrm{J}}$, for optical-pumping the metastable ${ }^{3} \mathrm{He}$ atoms by means of the high finesse external cavity with BiBO crystal. We obtained the second harmonic power of $380 \mathrm{~mW}$ with the maximum conversion efficiency of $56 \%$. To the best of our knowledge, it is the most efficient frequency-doubling based on BiBO crystal recently reached at around $390 \mathrm{~nm}$. In addition, the output power was stable enough to scan the frequency and to perform the spectroscopy.
\end{abstract}

DOI: $10.2961 / \mathrm{jlmn} .2009 .03 .0015$

Keywords: MRI, nuclear spin polarization, ${ }^{3} \mathrm{He}$, frequency-doubling, BiBO crystal,

\section{Introduction}

Porous materials are ubiquitous in nature. For example, there are granular materials, foams, ceramics, reservoir rocks, and animal lungs and sinuses. We can also fabricate a structure by such artificial means as laser-processing. In order to investigate the surface and three-dimensional structure of such porous materials, a hyperpolarized gas has been extensively researched by the characteristics of "Fast gas diffusion" and "Long spin polarization lifetime" which the hyperpolarized noble gas has. In short, proving the nuclear magnetic resonant signals the hyperpolarized noble gas emitting under a magnetic field, we can examine the surface structure of porous media with high resolution. Actually, Imaging of porous has been already obtained using the laser-polarized noble gas such as ${ }^{3} \mathrm{He}$ or ${ }^{129} \mathrm{Xe}$ [1] even at low field, though additional research is needed. Then, we have been focusing on hyperpolarized ${ }^{3} \mathrm{He}$ (helium-3), because it is less soluble in water and the polarized ${ }^{3} \mathrm{He}$ emits the strongest NMR signal in any other noble gases. Due to insoluble in water, the gas will be adjusted to the imaging of human lungs. Also, because of strongest NMR signal, we can obtain the higher resolution in taking the MRI scans even at the very lower field ( 10G) [2]. In order to polarize the nuclear spin of any atoms efficiently, we generally must optical-pump them via the optical resonance line, but the resonant light source in the vacuum and extreme UV region is needed for optical pumping of noble gases, and cannot be easily obtained. Therefore, we have been focusing on metastability exchange optical pumping (MEOP), which involves the metastable-state noble gas excited in an electric discharge and laser excitation.

We aimed to investigate the effect of the optical transition $2^{3} \mathrm{~S} \rightarrow 3^{3} \mathrm{P}$ (whose transition wavelength is $389 \mathrm{~nm}$ ) of the ${ }^{3} \mathrm{He}$ atom [3]. Therefore, we took the polarization was depended on the intensity of pumping light into account, and developed a very efficient 389-nm coherent light source and then have demonstrated the performance of the robust external cavity. This light source in the ultra-violet region makes use of the second harmonic ( $\mathrm{SH}$ ) generation in external cavity of continuous-wave mode-locked Ti:Sapphire laser with a nonlinear optical crystal, BiBO $\left(\mathrm{BiB}_{3} \mathrm{O}_{6}\right)$. Now, it is well known that $\mathrm{LiB}_{3} \mathrm{O}_{5}(\mathrm{LBO})$ [4] and $\beta-\mathrm{BaB}_{2} \mathrm{O}_{4}$ (BBO) [5] have been successfully developed and that they generate the second harmonic light highly efficiently at the UV region. However, the BiBO crystal has a larger effective nonlinear coefficient than LBO and $\mathrm{BBO}$. As a result, the theoretical single-pass conversion efficiency of $\mathrm{BiBO}$ is much larger than those of LBO and $\mathrm{BBO}$, even though the angular acceptance of $\mathrm{BiBO}$ is higher than that of $\mathrm{BBO}$ but lower than that of $\mathrm{LBO}$, and the walk-off angle in $\mathrm{BiBO}$ is lower than in $\mathrm{BBO}$ but higher than in LBO.

\section{Experimental setup}

Figure 1 shows a schematic of the setup for frequency doubling with the BiBO crystal. The light source is provided from a single-mode $\mathrm{cw}$ Ti:Sapphire laser, which is smaller than the linewidth of $50 \mathrm{kHz}$. Mode-Matching lens $(\mathrm{F}=300 \mathrm{~mm})$ coupled the collimated beam into the external enhancement cavity, which is a bow-tie ring resonator with two curved mirrors and two planar mirrors. The planar input coupler has a reflectivity of $99.3 \%$, while each of the three other mirrors has a reflectivity of $99.9 \%$ for the fundamental light. The reflectance of M1 was chosen in order to satisfy the condition of optical impedance matching. The output coupling mirror M3 is coated dichromatically, so that it can transmit $93.7 \%$ of the power of $\mathrm{SH}$ light. After leaving the cavity, the SH light is collimated in 


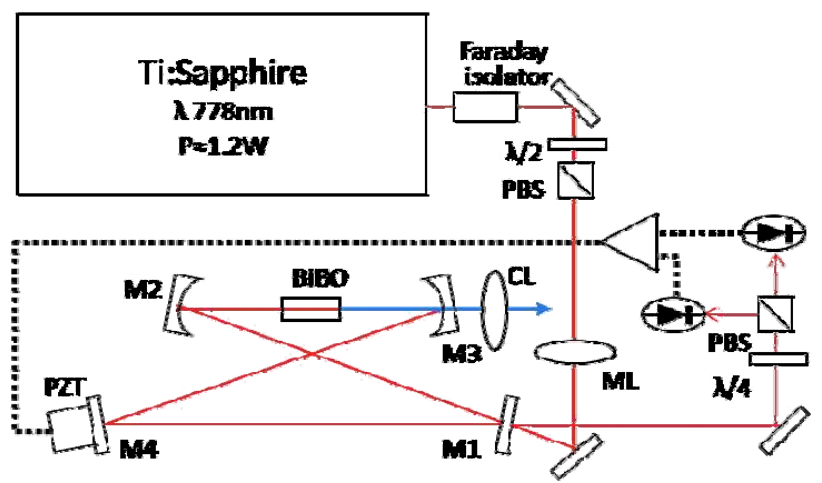

Fig. 1 Schematic of experimental setup for frequency doubling with BiBO crystal. PBS, polarizing beam splitter. ML, mode-matching lens. BS, beam splitter. CL, cylindrical lens.

the vertical planes by cylindrical lens, which results in about 9\% Fresnel's losses, to compensate the different beam divergences induced by walk-off in the nonlinear crystal. Inside the cavity, a $15-\mathrm{mm}$ bismuth-triborate (BiBO) crystal, which has an anti-reflection coating with a transmission of $99.8 \%$ for the SH light and $99.96 \%$ for the fundamental light, is placed in the focus of the curved mirrors M2 and M3. The curved mirrors have radii of $100 \mathrm{~mm}$, which is able to result in a minimum beam waist $\omega_{0}$ of $35 \mu \mathrm{m}$ inside the BiBO crystal. The beam waist size of the fundamental light was required to maximize the BoydKleinman focusing function [6], so the optical arrangement of the mirrors and the crystal was determined by the ABCD matrix. Here, this Boyd-Kleinman focusing function $\mathrm{h}(\mathrm{B}, \xi)$ describes the influence of the walk-off parameter B and the focusing parameter $\xi$ and is defined as

$$
h(B, \xi)=\frac{\pi^{2}}{\xi} \frac{1}{4 \pi^{2}} \iint_{-\xi}^{\xi} \frac{\exp \left[-B^{2}\left(\tau-\tau^{\prime}\right) / \xi\right]}{(1+i \tau)\left(1-i \tau^{\prime}\right)} d \tau \cdot d \tau^{\prime}
$$

In this experiment, $B$ equals 5.38 and the optimum BoydKleinman focusing function $\mathrm{h}(\mathrm{B}, \xi)$ is derived as 0.128 at $\xi=1.48$. Also, the BiBO crystal was cut for the collinear critical Type-I phase-matching direction $(\theta, \varphi)=\left(149.3^{\circ}\right.$, $90^{\circ}$ ), and a relatively large theoretical effective nonlinear optical coefficient, $\mathrm{d}_{\mathrm{eff}} 3.1 \mathrm{pm} / \mathrm{V}$, was obtained [7]. On the basis of the Boyd-Kleinman theory [6], we estimated the single-pass conversion efficiency to be $4.1 \times 10^{-4} / \mathrm{W}$. The optical arrangement of the mirrors and crystal was determined by the ABCD matrix. Mirror M4 is mounted on a piezoelectric transducer (PZT) and is controlled by a servo loop to stabilize the cavity length. The error signals were generated with a differential amplifier and feedback to PZT driver through servo electronics by the Hänsch-Couillaud method [8].

\section{Results and Discussion}

With the experimental setup described above, we can estimate the output power of SH generation by the equation [9]

$$
\sqrt{\mathrm{P}_{2 \omega}}=\frac{4 P_{\omega, \text { in }}\left(1-R_{c}\right) \sqrt{\gamma_{2 \omega}}}{\left[2-\sqrt{R_{c}}\left(2-\alpha_{1}-\sqrt{\gamma_{2 \omega} \mathrm{P}_{2 \omega}}\right)\right]^{2}}
$$

where $\mathrm{P}_{\omega, \text { in }}, \mathrm{P}_{2 \omega}, \mathrm{R}_{c}$, and $\alpha_{1}$ are the fundamental incident power just before the input coupler M1, the intrinsic power of the SH generation, the reflectance of M1, and the linear absorption of the external cavity, respectively. Figure 2

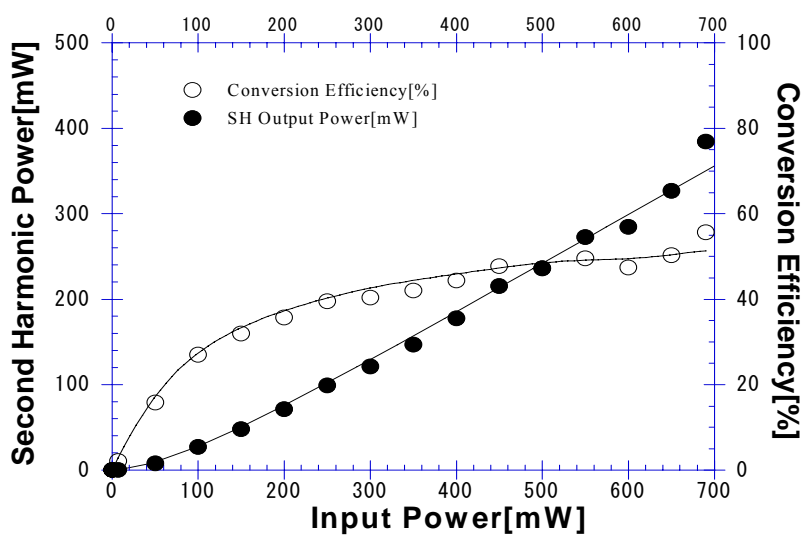

Fig. 2 Plots of generated second-harmonic output power in the cavity and the conversion efficiency as a function of incident fundamental power. Solid curve, fitted by Eq.(2)

shows the generated $\mathrm{SH}$ output power in the cavity as a function of the incident fundamental power. We obtained the SH power of $360 \mathrm{~mW}$ with a conversion efficiency of $52 \%$. The conversion efficiency is defined as the measured $\mathrm{SH}$ output power from the cavity, which is taking into account the 9\% Fresnel's loss of the cylindrical lens, divided by the incident fundamental power. The output power corresponds to the generation of $380 \mathrm{~mW}$ in the cavity, taking into account Fresnel's loss of the cylindrical lens, the transmissions of the BiBO and the output coupler. This result shows an overall intrinsic conversion efficiency of $56 \%$ from the input power into the generated power, which is the best result obtained, to our knowledge, for $\mathrm{SH}$ generation with a $\mathrm{BiBO}$ external cavity at around $389 \mathrm{~nm}$, even at the relatively low input power of $690 \mathrm{~mW}$. Also, these plots of the SH power show a nonlinear feature in accordance with the nonlinear optical phenomena. Then, we fitted a curve to our plots, like those shown in Fig. 2, using the equation (2). This fitting provides information about $\gamma_{2 \omega}$ and $\alpha_{1}$. In this way, $\gamma_{2 \omega}=2.3 \times 10^{-4} / \mathrm{W}$ and $\alpha_{1}=0.63 \%$ are inferred. This value of $\gamma_{2 \omega}$ was about $55 \%$ of the theoretical efficiency.

Using the obtained $\gamma_{2 \omega}$ and $\alpha_{1}$, enhancement factor $\varepsilon$ for the incident radiation in the cavity can be estimated using

$$
\varepsilon=\frac{P_{e}}{P_{\omega, \text { in }}}=\frac{1-R_{c}}{\left(1-\sqrt{\left.R_{c}\left(1-\alpha_{l}\right)\left(1-\sqrt{\gamma_{2 \omega} P_{2 \omega}}\right)\right)^{2}}\right.}
$$

where $P_{e}$ is the enhanced fundamental power at resonance. The enhancement factor, which is shown in Fig.3, is around 60 when the maximum incident fundamental power is $690 \mathrm{~mW}$. This value decreases with increasing fundamental power, because the nonlinear cavity loss increases. Figure 3 also shows the overall intracavity reflectance R, which is defined as $R=P_{r} / P_{\omega, \text { in }}$, where $P_{r}$ is the fundamental light intensity reflected off $\mathrm{M} 1$ at resonance. Only when mode matching and impedance matching are perfect, virtually no light will be reflected off M1 [10]. However, a large fraction of $P_{\omega, \text { in }}$, which is typically $50 \sim 40 \%$ in our experiment, is coming off M1. This mismatch may arise from the astigmatism of the laser beam. Therefore, the beam profile cannot be beam-formed perfectly into the beam diameter required in theory. As a result, it induced about $55 \%$ discrepancy of $\gamma_{2 \omega}$ between the estimated sin- 


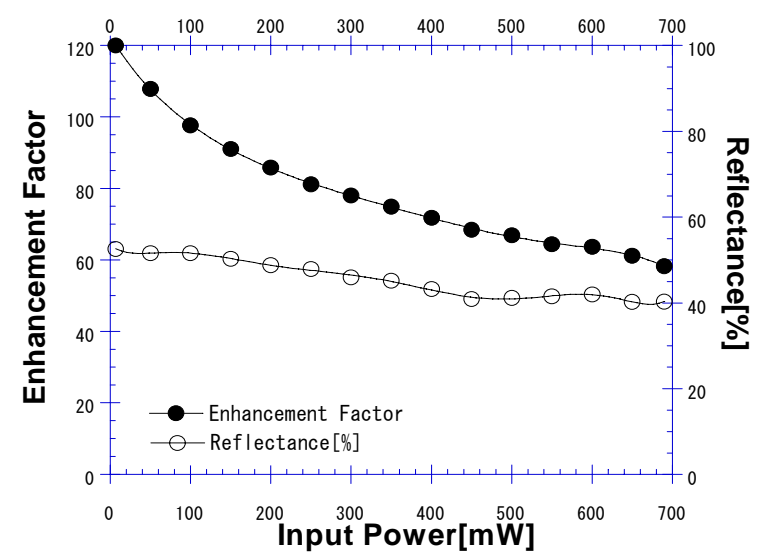

Fig. 3 Plots of estimated enhancement factor $\boldsymbol{\varepsilon}$ and the overall intracavity reflectance $\mathrm{R}$ as a function of incident fundamental power.

gle-pass conversion efficiency from the measurement and the theoretical efficiency.

The imperfect coating of the optical devices and the nonlinear depletion loss of the fundamental light power induce the linear loss chiefly. The nonlinear loss in the cavity arises from the absorption and/or scattering loss inside the $\mathrm{BiBO}$ crystal at the fundamental wavelength, where the absorption loss means that the fundamental power is converted into the SH light. It is obtained with $\alpha_{n l}=\gamma_{2 \omega} \cdot \varepsilon \cdot P_{\omega, \text { in }}$

Therefore, the nonlinear loss behaves similarly to as the $\mathrm{SH}$ power. At the incident power of $690 \mathrm{~mW}$, the linear and nonlinear intracavity losses are $0.63 \%$ and $0.93 \%$, respectively, whereas the total intracavity loss is $1.56 \%$.

The estimation of total cavity loss gives the linewidth for the cavity. The cavity finesse is given by the total cavity absorption as [11]

$$
F=\frac{\pi \sqrt{R_{c}}}{1-R_{c}} \approx \frac{2 \pi}{\alpha_{\text {total }}+T_{c}}
$$

Finesse of interferometer is calculated from the reflectance of an input coupler, but finesse of an external cavity should be estimated from the losses in the cavity with the righthandside of Eq.(5). Then, finesse can lead to the linewidth of the fundamental radiation for the cavity. The linewidth is given by

$$
\Delta \omega=\frac{F S R}{F}
$$

where FSR is the free spectral range. We obtained the cavity finesse of 274 at the incident power of $690 \mathrm{~mW}$. Then, we obtained the cavity linewidth of $1.76 \mathrm{MHz}$ and the free spectral range of $482 \mathrm{MHz}$ because the cavity length is 622 $\mathrm{mm}$. The obtained cavity linewidth still exceeds by far the laser linewidth. This allows to further increase the finesse of the enhancement cavity by reducing the roundtrip losses.

Figure 4 shows the stability of the developed SH light. When the fundamental frequency was scanned for the range of $20 \mathrm{GHz}$ around the wavelength of $778 \mathrm{~nm}$, the generated SH frequency was followed to the fundamental smoothly. During scanning, the fractional fluctuation of $3.1 \%$ in the generated SH light was measured. The interruption of the plots on the graph shown comes from a light source only when the Ti:Sapphire light source is unlocked.
If the light source was locked again, then the SH light was generated automatically at the same intensity.

\section{Conclusion}

We constructed an external high finesse cavity with a BiBO nonlinear crystal for laser enhancement, and have demonstrated very efficient frequency-doubling for a single frequency of 389-nm from a 778-nm Ti:Sapphire laser with a high conversion efficiency of $56 \%$ at the $\mathrm{SH}$ generation of $380 \mathrm{~mW}$ in the cavity. Considering the fact that our measured single-pass conversion efficiency reached only $55 \%$ of the estimated maximum value because of the mode-mismatch and/or impedance matching, if we improved the mismatches, then the achieved overall conversion efficiency of more than $50 \%$ would be indicated. The developed light source is extremely stable for many hours and can be frequency-stabilized to the transition within tens of GHz. Consequently, this laser system appears to be suitable for spectroscopy and for optical pumping of the nuclear spin polarization of ${ }^{3} \mathrm{He}$ atoms.

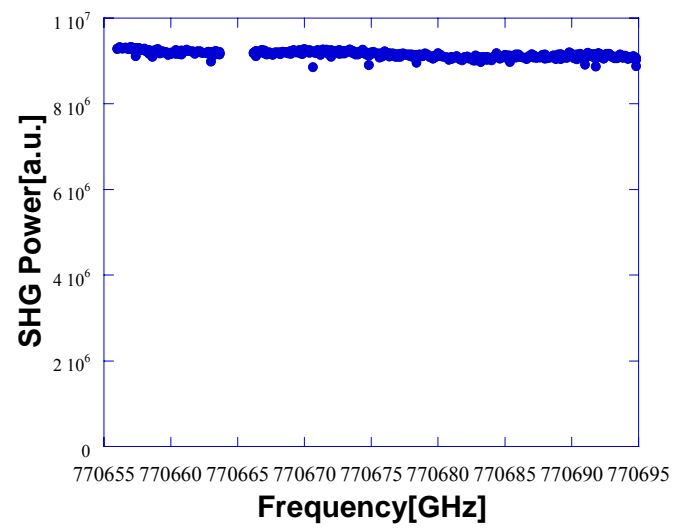

Fig. 4 Plots of the intensity of the second harmonic light for $40 \mathrm{GHz}$.

\section{References}

[1] G. P. Wong, et al.: J. Magn. Res. 141 (1999) 217

[2] J. M. Wild, et al.: Magn. Reson. Med. 52, (2004) 673.

[3] M. Fred, F. S. Tomkins, J.K. Brody, and M. Hamermesh, Phys. Rev. 82, (1951) 406.

[4] H. Kumagai, Y. Asakawa, T. Iwane, K. Midorikawa, and M. Obara, Appl. Opt. 42, (2003) 1036.

[5] J. D. Bhawalkar, Y. Mao, H. Po, A. K. Goyal, P. Gavrilovic, Y. Conturie, and S. Singh, Opt. Lett. 24, (1999) 823.

[6] G. D. Boyd and D. A. Kleiman, J. Appl. Phys. 39 (1968) 419.

[7] M. Ghotbi, and M. Ebrahim-Zadeh, Opt. Express. 12 (2004) 6002.

[8] T. W. Hänsch, and B. Couillaud, Opt. Commun. 35 (1980) 441.

[9] E. S. Polzik and H. J. Kimble, Opt. Lett. 16 (1991) 1400 .

[10] E. Jurdik, J. Hohlfeld, A. F. van Etteger, A. J. Toonen, W. L. Meerts, H. van Kempen, and Th. Rasing, JOSA B, 19, (2002) 1660.

[11]A. E. Siegman, "Lasers", (University Science Books, Mill Valley, California, 1986) pp. 435-436.

(Received: July 6, 2009, Accepted: November 24, 2009) 